www.nature.com/pj

\title{
Static partial scattering functions for linear and ring random copolymers
}

\author{
Hitoshi Endo and Mitsuhiro Shibayama
}

The static partial scattering functions for linear and ring random copolymers of type A-B are investigated. In the case of random distribution of the different kinds of monomers, analytic forms can be derived. Monte Carlo simulations are used to make evaluations on biased distributions of the different kinds of monomers. Results clearly show that the number, fraction and distribution of monomers have significant effect on the scattering intensities at $Q \times R_{g}>1$, where $Q$ is the magnitude of the scattering vector and $R_{g}$ is the radius of gyration of the polymer. The scattering function in molten state is also calculated on the basis of random phase approximation.

Polymer Journal (2010) 42, 157-160; doi:10.1038/pj.2009.325; published online 23 December 2009

Keywords: monte carlo simulation; partial scattering function; random copolymer; random phase approximation; ring polymer; small-angle scattering

\section{INTRODUCTION}

Development of a variety of polymers with specific molecular architecture, for example block and graft copolymers (for recent reviews on block and graft copolymer, see Hamley ${ }^{1}$ and Lodge ${ }^{2}$ ), star polymer (for recent reviews on star polymer, see Mishra ${ }^{3}$ and Grest $^{4}$ ), dendrimer, ${ }^{5}$ polyrotaxane ${ }^{6}$ and so on, has been the mainstream in polymer science for advanced applications (for recent reviews on designed polymerization, see $\operatorname{Hatada}^{7}$ and $\operatorname{Odian}^{8}$ ). Therefore, an investigation of the static configuration of these polymers is fundamental to the field and has been of intensive interest.

Historically, Flory ${ }^{9}$ first predicted that the static configuration of linear polymers on the melt obeys the Gaussian statistic, which was proved by small-angle neutron scattering with protonated polymers in a deutrated polymer matrix. ${ }^{10}$ Since then, many types of polymers have been identified by their static structures with the small-angle neutron scattering technique, and the contemporary theoretical progress in small-angle scattering has been of importance. ${ }^{11}$

In this study, the static scattering contributions from different kinds of monomer units in linear and ring random copolymers, which have hardly been given attention, are discussed. We assume random copolymers consisting of two different kinds of monomer units, namely monomer- $A$ and monomer- $B$. The static partial scattering functions of the monomer- $A$ component, that is, the scattering contribution from monomer- $A$ units, in linear and ring copolymers are derived on the basis of Gaussian statistics. Monte Carlo simulation is applied to evaluate the array of monomers. The scattering function of the molten random copolymers of type $A-B$ is also discussed.

\section{SCATTERING THEORY}

Linear random copolymer

Let us begin with the Debye function for the linear Gaussian polymer consisting of $N$ segments with the segment length $b,{ }^{12}$ that is,

$$
\begin{aligned}
S_{\text {Debye }}(Q) & =\frac{1}{N^{2}} \int_{0}^{N} \mathrm{~d} m \int_{0}^{N} \mathrm{~d} n \exp \left\{-\frac{Q^{2} b^{2}}{6}|m-n|\right\} \\
& =\frac{2}{Q^{4} R_{g l}^{4}}\left\{\exp \left(-Q^{2} R_{g l}^{2}\right)-1+Q^{2} R_{g l}^{2}\right\}
\end{aligned}
$$

with the magnitude of scattering vector $Q(=4 \pi \sin (\theta / 2) / \lambda$, scattering angle $\theta$, wavelength of incident beam $\lambda$ ) and the radius of gyration of the linear polymer $R_{g l}$ given by

$$
R_{g l}=\sqrt{\frac{N b^{2}}{6}}
$$

In the above description, the total length $L$ of the linear polymer can be defined as $L=N b$.

As the first step, Equation (1) is discretized with the interval $l_{0}$, namely,

$$
\begin{aligned}
& S_{\text {Debye }}^{\text {Linear }}(Q)=\frac{1}{N^{2}} \sum_{i=0}^{n_{0}-1} \sum_{j=0}^{n_{0}-1} \\
& \int_{i_{0} \cdot l_{0}}^{\left(i_{0}+1\right) \cdot l_{0}} \mathrm{~d} m \int_{j_{0} \cdot l_{0}}^{\left(j_{0}+1\right) \cdot l_{0}} \mathrm{~d} n \exp \left\{-\frac{Q^{2} b^{2}}{6}|m-n|\right\}
\end{aligned}
$$




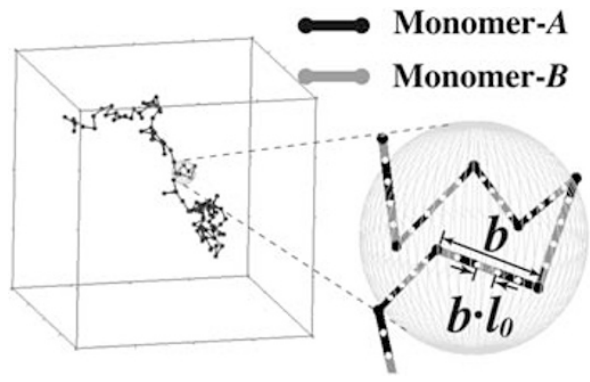

Figure 1 Schematic picture of a random copolymer consisting of a random sequence of $50 \%$ monomer- $A$ and $50 \%$ monomer- $B$, where the segment length is $b$ and the monomer length is $b \times 10$. The polymer is generated by 3D-random walks with 100 steps.

where $i$ and $j$ are natural numbers and $n_{0}$ is related to the interval $l_{0}$ as $l_{0}=\mathrm{N} / n_{0} . n_{0}$ roughly corresponds to the polymerization degree (the number of monomer units within the single polymer chain) by assuming that the lengths of monomer- $A$ and monomer- $B$ are the same. In this case, $b \times l_{0}$ is equivalent to the length of the single monomer unit. In our model, one segment of a Gaussian chain may hypothetically contain a number of monomers. In the case of flexible polymers, $l_{0}$ is very close to unity, and $l_{0}<1$ corresponds to semiflexible or rigid polymers. Figure 1 schematically depicts the model of a linear random copolymer.

To reflect the distribution of monomer- $A$ units, Equation (3) is modified as follows

$$
\begin{aligned}
& S_{\text {Debye }}^{\text {Linear }}(Q)=\frac{1}{N^{2}} \sum_{i=0}^{n_{0}-1} \sum_{j=0}^{n_{0}-1} A_{m}(i) A_{n}(j) \\
& \int_{i_{0} \cdot l_{0}}^{\left(i_{0}+1\right) \cdot l_{0}} \mathrm{~d} m \int_{j_{0} \cdot l_{0}}^{\left(j_{0}+1\right) \cdot l_{0}} \mathrm{~d} n \exp \left\{-\frac{Q^{2} b^{2}}{6}|m-n|\right\}
\end{aligned}
$$

where $A_{m, n}(k)$ is a $n_{0}$-dimensional vector defined by

$$
A_{m, n}(k)=\left\{\begin{array}{l}
1 \text { (monomer }-A \text { exist }) \\
0 \text { (monomer }-B \text { exist })
\end{array}\right.
$$

for the $k$-th interval. The ensemble average $\left\langle A_{m}(i) \cdot A_{n}(j)\right\rangle$ is given by

$$
\left\langle A_{m}(i) \cdot A_{n}(j)\right\rangle=\left\{\begin{array}{l}
\Phi_{\mathrm{A}}(\text { for } i=j) \\
\Phi_{\mathrm{A}}^{2}(\text { for } i \neq j)
\end{array}\right.
$$

with the length fraction of the monomer- $A$ component, $\Phi_{A}$.

In the case of a purely random process for the distribution of monomers, we can substitute $\left\langle A_{m}(i) \cdot A_{n}(j)\right\rangle$ for $A_{m}(i) \cdot A_{n}(j)$ in Equation (4), and in this condition, an analytic form of Equation (4) can be obtained as follows

$$
\begin{aligned}
S_{\text {Discr }}^{\text {Linear }}(Q)= & \frac{2}{x^{2}}\left[\exp (-x)-1-n_{0} \exp \left(-\frac{x}{n_{0}}\right)+n_{0}\right. \\
& \left.+\left\{n_{0} \exp \left(-\frac{x}{n_{0}}\right)-n_{0}+x\right\} / \Phi_{A}\right] / N_{l}
\end{aligned}
$$

with $x=Q^{2} R_{g l}{ }^{2}$ and $N_{l}=1+\left(1-\Phi_{A}\right) /\left(\Phi_{A} n_{0}\right)$.

\section{Ring random copolymer}

The above treatment can be applied to the ring polymer. On the basis of Gaussian statistics, the form factor of the ring polymer is given by ${ }^{13}$

$$
\begin{aligned}
S_{\text {Ring }}(Q) & =\frac{1}{N^{2}} \int_{0}^{N} \mathrm{~d} m \int_{0}^{N} \mathrm{~d} n \exp \left\lfloor-\frac{Q^{2} b^{2}}{6}\left\{|m-n|-\frac{(m-n)^{2}}{N}\right\}\right\rfloor \\
& =\frac{\sqrt{2}}{Q R_{g r}} \cdot D\left(\frac{Q R_{g r}}{\sqrt{2}}\right)
\end{aligned}
$$

where $D(u)$ is the Dawson function, that is, $D(u)=$ $\exp \left(-u^{2}\right) \int_{0}^{u} \exp \left(t^{2}\right) \mathrm{d} t$, and $R_{g r}$ is the radius of gyration for the ring polymer defined by

$$
R_{g r}=\sqrt{\frac{N b^{2}}{12}}
$$

Thereafter, the discretization and summation for the purely random process of the distribution of monomers are executed for the length fraction of the monomers, $\Phi_{A}$, in the same way as in Equations (3)-(7), and after some derivations, we obtain

$$
\begin{aligned}
S_{\text {Discr }}^{\text {Ring }}(Q)= & \frac{1}{N^{2}} \sum_{i=0}^{n_{0}-1} \sum_{j=0}^{n_{0}-1}\left\langle A_{m}(i) A_{n}(j)\right\rangle \int_{i_{0} \cdot l_{0}}^{\left(i_{0}+1\right) \cdot l_{0}} \mathrm{~d} m \int_{j_{0} \cdot l_{0}}^{\left(j_{0}+1\right) \cdot l_{0}} \mathrm{~d} n \\
& \times \exp \left[-\frac{Q^{2} b^{2}}{6}\left\{|m-n|-\frac{(m-n)^{2}}{N}\right\}\right] \\
= & \frac{1}{2 x}\left[\left\{n_{0}-\sqrt{2 x}\left(n_{0}-2\right) \cdot D\left(\sqrt{\frac{x}{2}}\right)-F\right\} / \Phi_{A}\right. \\
& \left.-\left\{n_{0}-\sqrt{2 x} \cdot n_{0} \cdot D\left(\sqrt{\frac{x}{2}}\right)-F\right\}\right] / N_{r}
\end{aligned}
$$

where

$$
\begin{aligned}
& F=\exp \left\{\frac{2 x}{n_{0}}\left(\frac{1}{n_{0}}-1\right)\right\} \\
& \times\left\{n_{0}-\sqrt{2 x}\left(n_{0}-2\right) \cdot D\left(\sqrt{\frac{x}{2}}\left(1-\frac{2}{n_{0}}\right)\right)\right\} \\
& \text { with } x=Q^{2} R_{g r}{ }^{2} \text { and } N_{r}=1+\left(1-\Phi_{A}\right) /\left(\Phi_{A} n_{0}\right) \text {. }
\end{aligned}
$$

\section{RESULTS AND DISCUSSION}

Behavior of $S_{\text {Discr }}^{\text {Linear }}(Q)$ and $S_{\text {Discr }}^{\text {Ring }}(Q)$

The effects of the parameters $\Phi_{A}$ and $n_{0}\left(=\mathrm{N} / l_{0}\right)$ on $S_{\text {Discr }}^{\text {Linear }}(Q)$ in Equation (7) are examined in Figure 2 and Figure 3. It is clearly seen that the normalized scattering function is hardly affected by $\Phi_{A}$ and $n_{0}$ for $Q R_{g l}<1$, whereas excess scattering intensity arises with decreasing $\Phi_{A}$ and/or $n_{0}$ for $Q R_{g l}>1$.

In Figure $4, S_{\text {Ring }}(Q)$ values given by Equation (8) are clarified with $S_{\text {Debye }}(Q)$ values given by Equation $(1)$, where $Q \times R_{g}$ for $S_{\text {Debye }}(Q)$ is multiplied by $\sqrt{2}$ to compare the shape of the curves. The essential difference between $S_{\text {Debye }}(Q)$ and $S_{\text {Ring }}(Q)$ is the curvature at $Q \approx 1 / R_{g}$, that is, the curvature of $S_{\text {Ring }}(Q)$ is greater than that of $S_{\text {Debye }}(Q)$. The dependence of $\Phi_{A}$ and $n_{0}$ on $S_{\text {Discr }}^{\text {Ring }}(Q)$ in Equation (10) is similar to $S_{\text {Discr }}^{\text {Linear }}(Q)$, that is, excess scattering is observed for $Q R_{g r}>1$ with decreasing $\Phi_{A}$ and/or $n_{0}$ as exhibited in Figure 4 .

\section{Effect of biased monomer distribution}

The effect of the distribution of monomers can be investigated by Monte Carlo simulation. We define the conditional probability that two monomer- $A$ units are successively placed as $\phi_{A A}$, as well as the conditional probability that a monomer- $A$ unit and a monomer- $B$ unit are successively placed as $\phi_{A B}$. Thereafter, $\Phi_{A}, \phi_{A A}$ and $\phi_{A B}$ are 


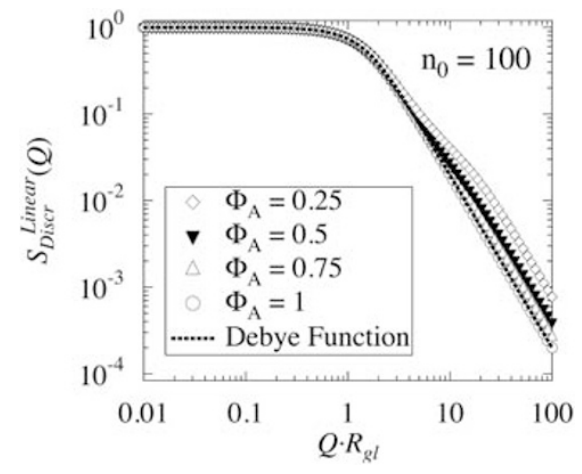

Figure 2 Normalized partial scattering functions for linear random copolymers given by Equation (7) with different $\Phi_{A}$ as a function of $Q R_{g}$ : $\Phi_{A}=0.25(\diamond) ; \Phi_{A}=0.5(\boldsymbol{\nabla}) ; \Phi_{A}=0.75(\triangle) ; \Phi_{A}=0.25(\bigcirc)$; the normalized Debye function given by Equation (1) (---). The fixed parameter is $n_{0}$ $(=100)$.

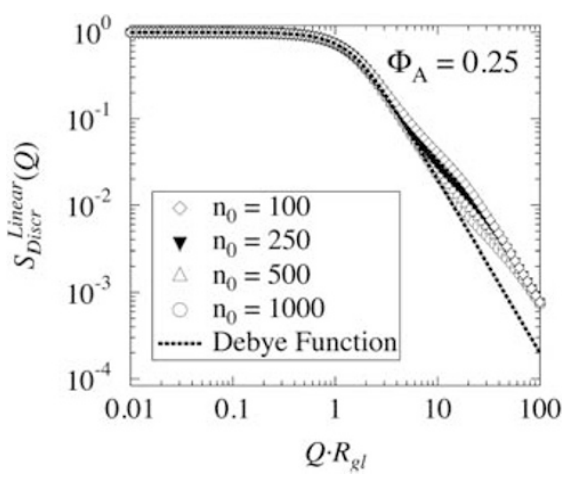

Figure 3 Normalized partial scattering functions for linear random copolymers given by Equation (7) with different $n_{0}$ as a function of $Q R_{g}$ : $n_{0}=100(\diamond) ; n_{0}=250(\boldsymbol{\nabla}) ; n_{0}=500(\triangle) ; n_{0}=1000(O)$; the normalized Debye function given by Equation (1) (---). The fixed parameter is $\Phi_{A}$ $(=0.25)$.

related as follows

$$
\phi_{A B}=\frac{\Phi_{A}}{1-\Phi_{A}}\left(1-\phi_{A A}\right)
$$

In this simple model, $\Phi_{A}<\phi_{A A}$ indicates that monomer- $A$ units tend to neighbor; similarly, $\Phi_{A}>\phi_{A A}$ indicates that monomer- $A$ units are distributed separately. $A_{m, n}(k)$ in Equation (4) is determined by generating random numbers $\phi_{k}$ between 0 and 1 as follows,

$$
A_{m, n}(k)=\left\{\begin{array}{l}
1\left(\text { for } \phi_{k} \leqslant \phi_{A A}\right) \\
0\left(\text { for } \phi_{k}>\phi_{A A}\right)
\end{array}\right.
$$

in the case of $A_{m, n}(k-1)=1$, and

$$
A_{m, n}(k)=\left\{\begin{array}{l}
1\left(\text { for } \phi_{k} \leqslant \phi_{A B}\right) \\
0\left(\text { for } \phi_{k}>\phi_{A B}\right)
\end{array}\right.
$$

in the case of $A_{m, n}(k-1)=0$.

In Figure 5, the results of Monte Carlo simulations by changing $\phi_{A A}$ with $n_{0}=100$ and $\Phi_{A}=0.25$ are exhibited. Sampling was repeated until the relative variation between $n$-th and $(n+1)$-th step became less than $10^{-5}$. Otherwise, $S_{\mathrm{MC}}^{\mathrm{Linear}}(Q)$ was the average of $10^{4}$ samplings. The partial scattering function, $S_{\mathrm{MC}}^{\text {Linear }}(Q)$, is affected only at $Q R_{g}>1$, and the additional scattering intensity arises with increasing $\phi_{A A}$. $S_{\mathrm{MC}}^{\mathrm{Linear}}(Q)$ with $\phi_{A A}=1$ coincides with the Debye function

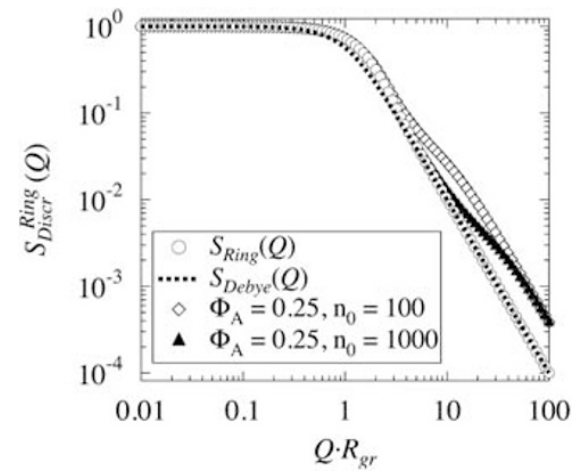

Figure 4 Normalized partial scattering functions for ring random copolymers given by Equation (10) with different $n_{0}$ and $\Phi_{A}$ as a function of $Q R_{g}$ : $\Phi_{A}=1$ $(\bigcirc)$; the normalized Debye function given by Equation (1) (---); $\Phi_{A}=0.25$ and $n_{0}=100(\diamond) ; \Phi_{A}=0.25$ and $n_{0}=1000(\boldsymbol{\Delta})$.

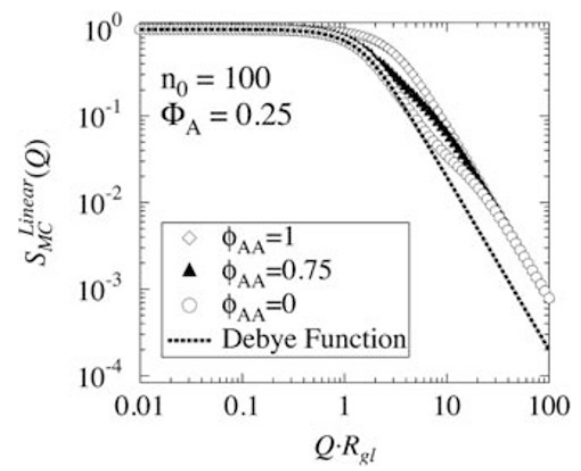

Figure 5 Normalized partial scattering functions for linear random copolymers obtained by Monte Carlo simulation with different $\phi_{A A}$ as a function of $Q R_{g}: \phi_{A A}=1(\diamond) ; \phi_{A A}=0.75(\mathbf{\Lambda}) ; \phi_{A A}=0(\bigcirc)$; the normalized Debye function given by Equation (1) (---). The fixed parameters are $n_{0}$ $(=100)$ and $\Phi_{A}(=0.25)$.

$S_{\text {Debye }}(Q)=2\{\exp (-x)-1+x\} / x^{2}$ with $x=Q^{2} R_{g}^{2} \Phi_{A}$. The results obviously confirm that the alignment of different kinds of monomers is also an import condition for the partial scattering function.

\section{Molten state}

The scattering function for molten copolymers can be calculated on the basis of random phase approximation (RPA).${ }^{14}$ For instance, static scattering functions for multiblock copolymers with various architectures in the molten state were obtained by RPA. ${ }^{15}$ In the case of the linear random copolymer of monomer- $A$ and monomer- $B$ with random monomer distribution, RPA leads to the scattering function as

$$
S_{\mathrm{RPA}}^{\mathrm{Linear}}=\frac{N}{S-2 \chi N}
$$

with

$$
S=\frac{S_{11}+2 S_{12}+S_{22}}{S_{11} \cdot S_{22}-S_{12}^{2}}
$$

where $N$ is the segment number and

$$
\begin{aligned}
& S_{11}=\Phi_{A}^{2} \cdot S_{\text {Discr }}^{\text {Linear }}\left(x, \Phi_{A}, n_{0}\right) \\
& S_{22}=\left(1-\Phi_{A}\right)^{2} \cdot S_{\text {Discr }}^{\text {Linear }}\left(x, 1-\Phi_{A}, n_{0}\right) \\
& S_{12}=\Phi_{A}\left(1-\Phi_{A}\right) \cdot S_{\text {Debye }}(x)
\end{aligned}
$$




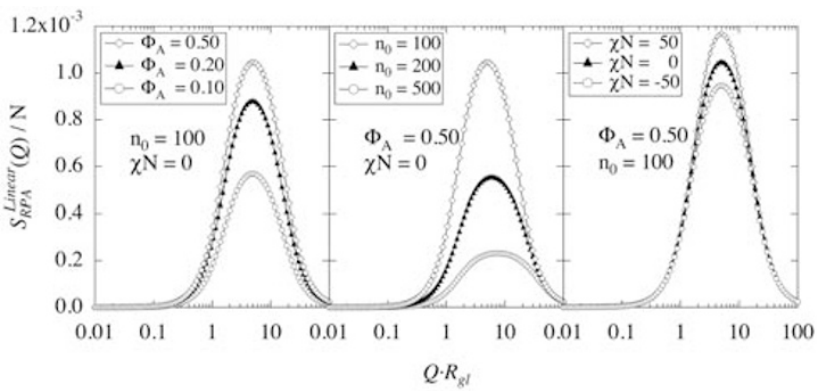

Figure 6 Scattering intensities for molten random linear copolymers of type $A-B$ given by Equations (15)-(17) with the monomer- $A$ fraction, $\Phi_{A}$, the monomer number, $n_{0}$, and the Flory-Huggins segment-segment interaction parameter, $\chi$, as a function of $Q R_{\mathrm{g}}$ : (left) $\Phi_{A}$ dependence by fixing $n_{0}=100$ and $\chi=0: \Phi_{A}=0.5(\diamond) ; \Phi_{A}=0.2(\boldsymbol{\Lambda}) ; \Phi_{A}=0.1(\bigcirc)$ : (center) $n_{0}$ dependence by fixing $\Phi_{A}=0.5$ and $\chi=0: n_{0}=100(\diamond) ; n_{0}=200(\boldsymbol{\Delta}) ; n_{0}=500(\bigcirc)$ : (right) $\chi$ dependence by fixing $\Phi_{A}=0.5$ and $n_{0}=100: \chi \mathrm{N}=50(\diamond) ; \chi \mathrm{N}=0$ $(\boldsymbol{\Delta}) ; \chi \mathrm{N}=-50(\bigcirc)$; with the segment number $N$.

with $x=Q^{2} R_{g l}{ }^{2}$, and $S_{\text {Debye }}(x)$ and $S_{\text {Discr }}^{\text {Linear }}\left(x, \Phi_{A}, n_{0}\right)$ are defined by Equations (1) and (7), respectively. The other parameters are the Flory-Huggins segment-segment interaction parameter, $\chi$, the length fraction of monomer- $A, \Phi_{A}$ and the number of monomer units, $n_{0}$. In Figure 6, the parametric calculations with Equations (15)-(17) are shown, where the tested parameters are $\Phi_{A}, n_{0}$ and $\chi$. In the case of $\Phi_{A}$ dependence, see Figure 6 left, peak heights were only affected, that is, the highest peak is given by $\Phi_{A}=0.50$ and the heights decreased with $\Phi_{A} \rightarrow 0$ and $\Phi_{A} \rightarrow 1$, whereas the peak widths and peak positions were the same for each $\Phi_{A}$. On the other hand, both the peak heights, peak widths and peak positions were evidently affected by different $n_{0}$ values as shown in Figure 6 center, where the peak heights were decreased with increasing $n_{0}$, and at the same time, the peak widths broadened and the peak positions shifted to a higher $Q$. The $\chi$ dependence was similar to the linear polymer blends, that is, the peak height with larger $\chi$ was higher than that of smaller $\chi$, and the peak width with larger $\chi$ was narrower than that of smaller $\chi$, as exhibited in Figure 6 right.

\section{Experimental feasibility}

The experimental observation of such behaviors is feasible, for example, by contrast matched or contrast variation neutron scattering experiments with partially deuterated random copolymers. ${ }^{11}$ In the case of small-angle neutron scattering (SANS), the observable highest$Q$ of the usual diffractometers with pinhole collimaters and a constant neutron wavelength is limited, for example, the limitation of SANS-U diffractometer at JRR-3 in Japan is $Q_{M A X} \approx 0.2 \AA^{-1}$ with $\lambda=7 \AA .{ }^{16}$ To examine the difference between a random copolymer and the corresponding homopolymer by SANS, the radius of gyration, $R_{g}$, (that is, the molecular weight) of the measured polymer should be absolutely large. For example, $R_{g}$ of polystyrene with molecular weight $40 \mathrm{~kg} \mathrm{~mol}^{-1}$ in the $\theta$-condition is $57 \AA^{17},{ }^{17}$ which gives $Q=0.175 \AA^{-1}$ for $Q \times R_{g}=10$. Therefore, we believe that experimental verification with a usual SANS diffractometer is possible. Future time of flight SANS diffractometers at the second generation spallation neutron sources will be certainly useful for the purpose, as diffractometers can cover a higher $Q$-range $\left(Q_{M A X} \sim 50 \AA^{-1}\right){ }^{18}$

\section{CONCLUSIONS}

The static partial scattering functions for linear and ring random copolymers were evaluated. In the case of random distribution of monomers, analytic forms were obtained. Monte Carlo simulation was used to verify the effects on biased distribution of the monomers. Results clearly showed that the number, fraction and distribution of monomers have a significant effect on the scattering intensities at $Q R_{g}>1$, which could be examined by scattering experiments.

In the case of molten state, scattering intensity was obtained by random phase approximation. The appearance of a single peak was numerically predicted, which has an analogy to block copolymers of type $A-B$. It was shown that the shape, height and position of the peak were affected by the three parameters, namely, fraction, number of monomers and the Flory-Huggins segment-segment interaction parameter.

Furthermore, the mathematical treatments and methodology achieved in the article can be applied for the detailed analyses of random copolymers of semiflexible non-Gaussian chains with the helical wormlike chain model, ${ }^{19}$ distribution of beaded molecules in polyrotaxane $e^{20,21}$ and so on by means of small-angle scattering.

\section{ACKNOWLEDGEMENTS}

We acknowledge the valuable discussions with Dr Ryuhei Motokawa at the Japan Atomic Energy Agency, Professor Takahiro Sato at Osaka University, and Mr Koichi Mayumi at the University of Tokyo.

1 Hamley, I. (ed.) Developments in Block Copolymer Science and Technology (Wiley, New York, 2004).

2 Lodge, T. P. Block copolymers: past successes and future challenges. Macromol. Chem. Phys. 204, 265-273 (2003).

3 Mishra, M. K. \& Kobayashi, S. (eds). Star and Hyperbranched Polymers (Marcel Dekker, New York, 1999).

4 Grest, G. S., Fetters, L. J., Huang, J. S. \& Richter, D. Star polymers: experiment, theory, and simulation. Adv. Chem. Phys. 94, 67-163 (1996).

5 Tomalia, D. A., Baker, H., Dewald, J., Hall, M., Kallos, G., Martin, S., Roeck, J., Ryder, J. \& Smith, P. A. New class of polymers: starburst-dendritic macromolecules. Polym. J. 17, 117-132 (1985)

6 Harada, A., Li, J. \& Kamachi, M. The molecular necklace: a rotaxane containing many threaded $\alpha$-cyclodextrins. Nature 356, 325-327 (1992).

7 Hatada, K., Kitayama, T. \& Vogl, O. (eds). Macromolecular Design of Polymeric Materials (Marcel Dekker, New York, 1997).

8 Odian, G. Principles of Polymerization 4th Edition (Wiley, New York, 2004).

9 Flory, P. Introduction to Polymer Chemistry (Cornell University Press, Ithaca, New York, 1967)

10 Ballard, D. G. H., Wignall, G. D. \& Schelten, J. Measurement of molecular dimensions of polystyrene chains in the bulk polymer by low angle neutron diffraction. Eur. Polym. J. 9, 965-969 (1973)

11 Higgins, J. S. \& Benoit, H. C. Polymers and Neutron Scattering (Clarendon Press, Oxford, 1994).

12 Debye, P. J. Molecular-weight determination by light scattering. Phys. Colloid Chem. 51, 18-32 (1947)

13 Casassa, E. F. Some statistical properties of flexible ring polymers. J. Polym. Sci. Part A 3, 605-614 (1965).

14 de Gennes, P. G. Scaling Concepts in Polymer Physics (Cornel1 University Press, Ithaca, New York, 1979).

15 Benoit, H. \& Hadziioannou, G. Scattering theory and properties of block copolymers with various architectures in the homogeneous bulk state. Macromolecules 21, 14491464 (1988).

16 Okabe, S., Karino, T., Nagao, M., Watanabe, S. \& Shibayama, M. Current status of the $32 \mathrm{~m}$ small-angle neutron scattering instrument, SANS-U. Nucl. Instrum. Methods Phys. Res. Sect A 572, 853-858 (2007).

17 Konishi, T., Yoshizaki, T., Saito, T., Einaga, Y. \& Yamakawa, H. Mean-square radius of gyration of oligo- and polystyrenes in dilute solutions. Macromolecules 23, 290-297 (1990).

18 Shinohara, T., Takata, S., Suzuki, J., Oku, T., Suzuya, K., Aizawa, K., Arai, M., Otomo, T. \& Sugiyama, M. Design and performance analyses of the new time-of-flight smaller-angle neutron scattering instrument at J-PARC. Nucl. Instrum. Meth. Phys. Res. Sect. A 600, 111-113 (2009).

19 Yoshizaki, T. \& Yamakawa, H. Scattering functions of wormlike and helical wormlike chains. Macromolecules 13, 1518-1525 (1980).

20 Mayumi, K., Osaka, N., Endo, H., Yokoyama, H., Sakai, Y., Shibayama, M. \& Ito, K. Concentration-induced conformational change in linear polymer threaded into cyclic molecules. Macromolecules 41, 6480-6485 (2008).

21 Mayumi, K., Endo, H., Osaka, N., Yokoyama, H., Nagao, M., Shibayama, M. \& Ito, K. Mechanically interlocked structure of polyrotaxane investigated by contrast variation small-angle neutron scattering. Macromolecules 42, 6327-6329 (2009). 